Vol. 42 (1990) [231-245]

\title{
ON THE ISOMETRY OF SURFACES PRESERVING THE LINES OF CURVATURE WENMAO YANG
}

In this paper we study the isometric deformations of surfaces in $E^{3}$, which preserve the lines of curvature. We call a surface $M$ and $L C$-surface if it admits a nontrivial deformation of this type. We distinguish three types of $L C$-surfaces, and obtain some new results about these three types of surfaces.

\section{INTRODUCTION}

Bryant, Chern and Griffiths [1] studied the isometric deformations of surfaces in 3dimensional Euclidean space $E^{3}$, which preserve the lines of curvature. They obtained a main result as follows:

THEOREM A. In the three-dimensional Euclidean space $E^{3}$ consider two pieces of surfaces $M, M^{*}$, such that (a) their Gaussian curvature is not zero and they have no umbilics; and (b) they are connected by an isometry $f: M \rightarrow M^{*}$ preserving the lines of curvature. Then $M$ and $M^{*}$ are in general congruent or symmetric. There are surfaces $M$, for which the corresponding $M^{*}$ is distinct relative to rigid motions. The Molding surfaces, and only these, are such surfaces belonging to a continuous family of distinct surfaces, which are connected by isometries preserving the lines of curvature (see $[\mathbf{1}]$, p.283).

In the present paper we shall study deeply the deformations. First of all, we have the following definition.

Definition. Let $M$ and $M^{*}$ be two surfaces in $E^{3}$ and $I$ and $I^{*}$ be the first fundamental forms of $M$ and $M^{*}$. Suppose a map $F: M \rightarrow M^{*}$ is a diffeormorphism, and

(a) $F$ is an $I$-isometry

$$
F^{*}\left(I^{*}\right)=I
$$

where $F^{*}$ represents $F$ 's cotangent map.

(b) $F$ preserves the lines of curvature.

Received 20 October 1989

The author would like to thank Professor Hyam Rubinstein for his help and encouragement.

Copyright Clearance Centre, Inc. Serial-fee code: 0004-9729/90 \$A2.00+0.00. 
In this case, we call $F$ an isometry preserving the lines of curvature, or an ' $L C$ isometry'. If a surface $M$ admits a non-trivial $L C$-isometry, we call $M$ an $L C$-surface.

Relative to the integrability conditions of a differential equation (see (1.26) and (1.27)), we can distinguish three types of $L C$-surfaces. We obtain some new results about the three types of $L C$-surfaces.

(1) Theorem A supposes that the Gaussian curvatures of the surfaces $M$ and $M^{*}$ are not zero. We shall prove that any developable surface (with Gaussian curvature zero) is an $L C$-surface of the first type (see Section 2, Theorem 1).

(2) An $L C$-surface of the second type is just a Molding surface which was studied in Theorem A. We shall get some new properties of the surface (see Section 3, Theorem 2, and Theorem 3 and its Corollary).

(3) We shall get a necessary and sufficient condition for a surface to be an $L C$-surface of the third type (see Section 4, Theorem 4).

\section{ISOMETRY PRESERVES THE LINES OF CURVATURE}

We study a piece of an oriented surface $M$ in 3-dimensional Euclidean space $E^{\mathbf{s}}$, and suppose it to be sufficiently differentiable and with no umbilic points. On $M$ there is a field of orthonormal frames $m e_{1} e_{2} e_{3}$, such that $m \in M$, where $e_{1}$ and $e_{2}$ are unit vectors along the principal directions of $M$ at $m$, and $e_{3}$ is the unit normal vector to $M$ at $m$. We have

$$
\begin{aligned}
& d m=\omega_{1} e_{1}+\omega_{2} e_{2}, \\
& d e_{i}=\omega_{i j} e_{j} \\
& \omega_{i j}+\omega_{j i}=0, \quad i, j=1,2,3, \\
& \omega_{13}=a \omega_{1}, \omega_{23}=c \omega_{2} .
\end{aligned}
$$

We assume $a>c$, where $a$ and $c$ are the two principal curvatures of $M$ at $m$. The mean curvature and the Gaussian curvature of $M$ are

$$
2 H=a+c, \quad K=a c .
$$

The structural equations of $M$ are

$$
\begin{aligned}
d \omega_{1} & =-\omega_{2} \wedge \omega_{12}, \quad d \omega_{2}=\omega_{1} \wedge \omega_{12}, \\
d \omega_{12} & =-K \omega_{1} \wedge \omega_{2}=-\omega_{13} \wedge \omega_{23}, \\
d \omega_{13} & =-\omega_{23} \wedge \omega_{12}, \quad d \omega_{23}=\omega_{13} \wedge \omega_{12}
\end{aligned}
$$


The connection form of $M$ is

$$
\omega_{12}=h \omega_{1}+k \omega_{2} \text {. }
$$

The differentials of the two principal curvatures $a$ and $c$ are

$$
\begin{aligned}
& d a=a_{1} \omega_{1}+a_{2} \omega_{2}, \\
& d c=c_{1} \omega_{1}+c_{2} \omega_{2} .
\end{aligned}
$$

Define two functions

$$
f=a-c>0, \quad g=a+c .
$$

Using (1.4), (1.9), (1.10) and (1.11), the structural equations (1.7) imply

$$
a_{2}=f h, \quad c_{1}=f k .
$$

Suppose $M^{*}$ is another surface in the space $E^{3}$. We shall denote the quantities pertaining to $M^{*}$ by the same symbols with star "*", for example $\omega_{i j}^{*}, a^{*}, c^{*}, \ldots$

Let the mapping $F: M \rightarrow M^{*}$ be an isometry from $M$ to $M^{*}$, and $m e_{1} e_{2} e_{3}$ and $m^{*} e_{1}^{*} e_{2}^{*} e_{3}^{*}$ be the fields of principal frames over $M$ and $M^{*}$, respectively.

Since $F$ is an isometry, we have

$$
\begin{aligned}
& \omega_{1}^{*}=\omega_{1} \cos \tau-\omega_{2} \sin \tau \\
& \omega_{2}^{*}=\omega_{1} \sin \tau+\omega_{2} \cos \tau
\end{aligned}
$$

where $\tau$ is an angle of rotation of the principal directions during the isometric deformation. Now we suppose that $F$ preserves the lines of curvature, so it preserves the principal directions; so the angle $\tau=0$, and from (1.13), we get

$$
\omega_{1}^{*}=\omega_{1}, \quad \omega_{2}^{*}=\omega_{2}, \quad \omega_{12}^{*}=\omega_{12} .
$$

Since Gaussian curvature is preserved under isometry, $a c=a^{*} c^{*}$, and we can let

$$
a^{*}=t a, \quad c^{*}=t^{-1} c, \quad t \neq 0 .
$$

The geometric interpretations of $t$ and $t^{-1}$ are as change-coefficients of the two principal curvatures $a$ and $c$, respectively. From (1.9), (1.11) and (1.14), we have

$$
\begin{aligned}
& h^{*}=h, \quad k^{*}=k, \\
& f^{*}=a^{*}-c^{*}=t a-t^{-1} c, \quad g^{*}=a^{*}+c^{*}=t a+t^{-1} c .
\end{aligned}
$$

From equation (1.12) when applied to $M^{*}$, we get

$$
(t a)_{2}=f^{*} h, \quad\left(t^{-1} c\right)_{1}=f^{*} k .
$$

Using (1.12) and (1.17) gives

$$
\begin{aligned}
& c t_{1}=t\left(1-t^{2}\right) a k, \\
& a t_{2}=-t^{-1}\left(1-t^{2}\right) c h .
\end{aligned}
$$

We consider two cases depending on whether $M$ is a developable surface or not. 
I. $M$ is a developable surface. In this case, $K=a c=0$. We suppose $a \neq 0, c=0$. From (1.18) we get

$$
k=0, \quad t_{2}=0
$$

and

$$
\omega_{13}=a \omega_{1}, \quad \omega_{2 s}=0 .
$$

Using (1.8) and (1.20), we have $d \omega_{1 s}=0$, so set $\omega_{1 s}$ as the total differential of the function $u_{1}$, a first parameter of $M$,

$$
\omega_{13}=d u_{1}, \quad \omega_{1}=a^{-1} d u_{1} .
$$

On the surface $M$ the set of $u_{1}$-curves (the curves along which $\omega_{2}=0$ ) is a family of lines of curvature which are not straight lines (because $a \neq 0$ ). But the other family of lines of curvature (the curves along $\omega_{1}=0$ ) are straight lines. We can choose the arclength of the straight lines as a second parameter $u_{2}$, so

$$
\omega_{2}=d u_{2} \text {. }
$$

Hence the differential equation of $t(1.18)$ or (1.19) becomes

$$
\frac{\partial t}{\partial u_{2}}=0
$$

its solution is

$$
t=t\left(u_{1}\right)
$$

Consequently, any developable surface $M$ is an $L C$-surface.

II. $M$ is not a developable surface. In this case, $K=a c \neq 0, a \neq 0, c \neq 0$. We rewrite (1.18) as

$$
\begin{aligned}
& t_{1}=t\left(1-t^{2}\right) a c^{-1} k, \\
& t_{2}=-t^{-1}\left(1-t^{2}\right) c a^{-1} h .
\end{aligned}
$$

From now on we assume $t_{2} \neq 1$, discarding the trivial case that $M^{*}$ is congruent or symmetric to $M$. Now we let

$$
\begin{aligned}
& \theta_{1}=a c^{-1} k \omega_{1}, \\
& \theta_{2}=c a^{-1} h \omega_{2},
\end{aligned}
$$


and

$$
T=t^{2} \neq 0,1
$$

so that $\left(1.18^{\prime}\right)$ can be written

$$
d T=2(1-T)\left(T \theta_{1}-\theta_{2}\right) .
$$

Taking exterior derivatives of (1.26) gives

$$
T\left(d \theta_{1}-2 \theta_{1} \wedge \theta_{2}\right)=d \theta_{2}-2 \theta_{1} \wedge \theta_{2}
$$

Equation (1.26) is the total differential equation satisfied by the square $T$ of the change-coefficient $t$ of the principal curvature $a$. Equation (1.27) is an integrability condition of (1.26). When solving (1.27) for $T$, on substituting into (1.26), we get the condition on the surface $M$, to be an $L C$-surface but not a developable surface.

Now let us distinguish three cases of $L C$-surfaces.

FirST TYPE. $\quad M$ is a developable surface, $K=0$, or $\theta_{1}=\theta_{2}=0$.

SECOND TYPE. $\quad M$ is not a developable surface, $K \neq 0$, and

$$
d \theta_{1}-2 \theta_{1} \wedge \theta_{2}=d \theta_{2}-2 \theta_{1} \wedge \theta_{2}=0 .
$$

Then (1.27) holds identically for all $T$, and (1.26) has a continuum of solutions, each depending on an arbitrary constant. Thus we have a one-parameter family of surfaces $L C$-isometric to $M$. This is just the case in Theorem A (see [1]).

THIRD TYPE. $\quad M$ is not a developable surface, $K \neq 0$, and (1.28) does not hold.

$$
d \theta_{1}-2 \theta_{1} \wedge \theta_{2} \neq 0, \quad d \theta_{2}-2 \theta_{1} \wedge \theta_{2} \neq 0 .
$$

Then from (1.27) we get $T$, inserting $T$ into (1.26). Thus we can obtain a single surface which is $L C$-isometric to $M$.

\section{LC -SURFACES OF THE FIRST TYPE - DEVELOPABLE SURFACES}

Suppose $M$ is a developable surface, and choose the frame field and the parameters $u_{1}, u_{2}$, so that (1.21) and (1.22) hold.

$$
\begin{aligned}
& \omega_{1}=a^{-1} d u_{1}, \quad \omega_{2}=d u_{2}, \quad a \neq 0, \\
& \omega_{13}=d u_{1}, \quad \omega_{23}=0, \quad c=0 \text {, }
\end{aligned}
$$


where the differential of the first parameter $u_{1}$ is the normal connection form $\omega_{13}$, and the second parameter $u_{2}$ is the arclength of the straight lines on the surface $M$. The change-coefficient $t$ of the principal curvatures as (1.23)

$$
t=t\left(u_{1}\right)
$$

is any function of $u_{1}$. From (1.15) and (1.14) we have some quantities of the surface $M^{*}$ :

$$
\begin{aligned}
a^{*} & =t\left(u_{1}\right) a, \quad c^{*}=0 \\
\omega_{1}^{*} & =\omega_{1}=a^{-1} d u_{1}, \quad \omega_{2}^{*}=\omega_{2}=d u_{2} \\
\omega_{12}^{*} & =\omega_{12}, \quad \omega_{13}^{*}=t\left(u_{1}\right) d u_{1}, \quad \omega_{23}^{*}=0 .
\end{aligned}
$$

From the above discussion we obtain the following theorem.

THEOREM 1. Any developable surface is an LC-surface of the first type. In other words, any developable surface can deform continuously to another developable surface by any isometry preserving the lines of curvature.

For the developable surface there exist three cases as follows:

(1). Cylinder M:

$$
\begin{aligned}
m(s, z) & =m(s)+z k, \\
m(s) & =x(s) i+y(s) j,
\end{aligned}
$$

where $O i j k$ is a frame in $E^{3}, m(s)$ a plane curve parametrised by its arclength $s$, and

$$
m^{\prime}=\alpha, \quad \alpha^{\prime}=\kappa \beta .
$$

Choosing a frame field over $M$, by

we have

$$
e_{1}=\alpha, \quad e_{2}=k, \quad e_{3}=-\beta,
$$

$$
\begin{aligned}
d m & =\omega_{1} e_{1}+\omega_{2} e_{2}, \\
\omega_{1} & =d s, \quad \omega_{2}=d z, \quad \omega_{12}=0, \\
\omega_{13} & =a \omega_{1}, \quad a=-\kappa \neq 0, \quad \omega_{2 s}=0 .
\end{aligned}
$$

Define the two parameters

$$
u_{1}=\int a(s) d s=-\int \kappa(s) d s, \quad u_{2}=z .
$$

Suppose $M^{*}$ is another cylinder which is $L C$-isometric to $M$. We have

$$
\omega_{13}^{*}=-\kappa^{*}\left(s^{*}\right) d s^{*}=t(s) \omega_{13}=-t(s) \kappa(s) d s \text {. }
$$

Hence

$$
\kappa^{*}\left(s^{*}\right) d s^{*}=t(s) \kappa(s) d s .
$$

This is the equation of an $L C$-isometry $F$. 
(2). Cone M:

$$
\begin{aligned}
m(s, v) & =v m(s), \\
m^{2}(s) & =1, \quad v>0
\end{aligned}
$$

where $m(s)$ is a curve on the unit sphere centered at the origin and parametrised by its arclength $s$. We have

$$
m^{\prime}=\alpha, \quad \alpha^{\prime}=\kappa \beta .
$$

Choose a frame field over $M$, by

$$
e_{1}=\alpha, \quad e_{2}=m, \quad e_{3}=\alpha \times m,
$$

and we have

$$
\begin{aligned}
d m & =\omega_{1} e_{1}+\omega_{2} e_{2} \\
\omega_{1} & =v d s, \quad \omega_{2}=d v \\
\omega_{12} & =v^{-1} \omega_{1}, \quad \omega_{13}=a \omega_{1}, \quad a=\kappa v^{-1}, \quad \omega_{23}=0 .
\end{aligned}
$$

The two parameters of $M$ are

$$
u_{1}=\int a \omega_{1}=\int \kappa(s) d s, \quad u_{2}=v .
$$

Suppose $M^{*}$ is another cone which is $L C$-isometric to $M$. We have the equation (2.5), too.

(3). Tangent surface $\mathrm{M}: m(s, \bar{v})=m(s)+\bar{v} \alpha(s), \quad \bar{v}>0$, where $m(s)$ is a nonplanar curve parametrised by its arclength $s$. We have

$$
\begin{aligned}
m^{\prime} & =\alpha, \quad \alpha^{\prime}=\kappa \beta, \quad \kappa \neq 0, \\
\beta^{\prime} & =-\kappa \alpha+\tau \gamma, \quad \gamma^{\prime}=-\tau \beta, \quad \tau \neq 0 .
\end{aligned}
$$

Choose a frame field of $M$, by

and we get

$$
e_{1}=\beta, \quad e_{2}=\alpha, \quad e_{3}=-\gamma
$$

or

$$
\begin{aligned}
d m & =(\kappa \bar{v} d s) \beta+(d s+d \bar{v}) \alpha \\
d m & =\omega_{1} e_{1}+\omega_{2} e_{2}, \\
\omega_{1} & =\kappa(v-s) d s, \quad \omega_{2}=d v, \\
\bar{v} & =v-s, \quad v>s . \\
\omega_{12} & =-\kappa d s, \quad \omega_{13}=a \omega_{1}, \quad \omega_{23}=0, \\
a & =-\tau \kappa^{-1}(v-s)^{-1} .
\end{aligned}
$$


The two parameters of $M$ are

$$
u_{1}=-\int \kappa(s) d s, \quad u_{2}=v .
$$

Let $M^{*}$ be another tangent surface which is $L C$-isometric to $M$. We have the equation of the $L C$-isometry $F$ as

$$
\tau^{*}\left(s^{*}\right)\left[\kappa^{*}\left(s^{*}\right)\left(v^{*}-s^{*}\right)\right]^{-1} d s^{*}=t(s) \tau(s)[\kappa(s)(v-s)]^{-1} d s .
$$

\section{LC-SURFACES OF THE SECOND TYPE - MOLDING SURFACES}

We now consider $L C$-surfaces of the second type. As is well known, [1] shows that these surfaces are Molding surfaces (see Theorem A), but in this section we obtain some new properties of these surfaces.

Suppose $Z$ is a cylinder and $C$ is a plane curve on some tangent plane $\pi$ of $Z$. The surface $M$ is the locus described by $C$ in space as the tangent plane $\pi$ rolls about $Z$. Such a surface $M$ is called a Molding surface (or $M$-surface).

TheOREM 2. An LC-surface of the second type is an M-surface.

This theorem is just Theorem $A$, and its proof is essentially contained in [1].

Proof I: We first prove $\sigma \cong h k=0$. According to the definition of an $L C$ surface of the second type, we have $K \neq 0, a \neq 0, c \neq 0$, so the two forms $\omega_{13}$ and $\omega_{23}$ are independent forms. We can choose them as fundamental forms for computation. Rewriting (1.24), in view of (1.4),

$$
\begin{aligned}
& \theta_{1}=c^{-1} k\left(a \omega_{1}\right)=k^{\prime} \omega_{13} \\
& \theta_{2}=a^{-1} h\left(c \omega_{2}\right)=h^{\prime} \omega_{23}
\end{aligned}
$$

where

$$
h^{\prime}=a^{-1} h, \quad k^{\prime}=c^{-1} k
$$

so that

$$
\omega_{12}=h^{\prime} \omega_{13}+k^{\prime} \omega_{23}
$$

Denote

$$
\sigma=h k=(a c)\left(h^{\prime} k^{\prime}\right)=K \sigma^{\prime}
$$

where

$$
\sigma^{\prime}=h^{\prime} k^{\prime}
$$


The structural equations (1.7) and (1.8) of the surface $M$ are

$$
\begin{aligned}
& d \omega_{12}=-* 1, \quad * 1 \cong \omega_{13} \wedge \omega_{23}, \\
& d \omega_{13}=h^{\prime} * 1, \quad d \omega_{23}=k^{\prime} * 1 .
\end{aligned}
$$

Inserting (3.1) into (1.27), in view of (3.2) and (3.5), we have

$$
\begin{aligned}
& \left(d h^{\prime}-\sigma^{\prime} \omega_{13}\right) \wedge \omega_{23}=0 \\
& \left(d k^{\prime}+\sigma^{\prime} \omega_{23}\right) \wedge \omega_{13}=0
\end{aligned}
$$

Using Cartan's Lemma from (3.8) implies

$$
\begin{aligned}
& d h^{\prime}=\sigma^{\prime} \omega_{13}+q^{\prime} \omega_{2 s}, \\
& d k^{\prime}=p^{\prime} \omega_{13}-\sigma^{\prime} \omega_{23},
\end{aligned}
$$

where $p^{\prime}$ and $q^{\prime}$ are two functions.

Taking exterior derivatives of (3.3), using (3.9) and (3.7) from (3.6) we obtain

$$
p^{\prime}-q^{\prime}=-\left(h^{\prime 2}+k^{\prime 2}+1\right) \text {. }
$$

We define a function $u$ by

$$
p^{\prime}+q^{\prime}=2 u
$$

Solving (3.10) and (3.11) for $p^{\prime}, q^{\prime}$

$$
\begin{aligned}
& p^{\prime}=u-\left(h^{\prime 2}+k^{\prime 2}+1\right) / 2, \\
& q^{\prime}=u+\left(h^{\prime 2}+k^{\prime 2}+1\right) / 2 .
\end{aligned}
$$

Taking derivatives of (3.5), in view of (3.9), we get

$$
d \sigma^{\prime}=\left(h^{\prime} p^{\prime}+k^{\prime} \sigma^{\prime}\right) \omega_{13}+\left(k^{\prime} q^{\prime}-h^{\prime} \sigma^{\prime}\right) \omega_{23} .
$$

Taking exterior derivatives of (3.9), using (3.13), we have

$$
\begin{aligned}
& \left(d q^{\prime}+2 h^{\prime} \sigma^{\prime} \omega_{13}\right) \wedge \omega_{23}=0 \\
& \left(d p^{\prime}+2 k^{\prime} \sigma^{\prime} \omega_{23}\right) \wedge \omega_{13}=0
\end{aligned}
$$

so

$$
\begin{aligned}
& d p^{\prime}=p^{\prime \prime} \omega_{13}-2 k^{\prime} \sigma^{\prime} \omega_{23}, \\
& d q^{\prime}=-2 h^{\prime} \sigma^{\prime} \omega_{13}+q^{\prime \prime} \omega_{23} .
\end{aligned}
$$


Taking derivatives of (3.10), using (3.9) and (3.15), we get

$$
\begin{aligned}
& p^{\prime \prime}=-2\left(k^{\prime} p^{\prime}+2 h^{\prime} \sigma^{\prime}\right), \\
& q^{\prime \prime}=2\left(h^{\prime} q^{\prime}-2 k^{\prime} \sigma^{\prime}\right) .
\end{aligned}
$$

Taking derivatives of (3.11), from (3.15) and (3.16), we get

$$
\begin{aligned}
d u= & {\left[k^{\prime}\left(-u+\frac{1}{2}\right)-\frac{1}{2} k^{\prime 3}-\frac{5}{2} h^{\prime} \sigma^{\prime}\right] \omega_{13} } \\
& +\left[h^{\prime}\left(u+\frac{1}{2}\right)+\frac{1}{2} h^{\prime 3}-\frac{5}{2} k^{\prime} \sigma^{\prime}\right] \omega_{23} .
\end{aligned}
$$

Taking exterior derivatives of (3.17), using (3.9), (3.13) and (3.17), we get

$$
\sigma^{\prime}=0 \quad \text { or } \quad \sigma=0 .
$$

We suppose

$$
h \neq 0, \quad k=0
$$

From (3.9) and (3.10), we have

$$
p^{\prime}=0, \quad q^{\prime}=1+h^{\prime 2}
$$

For this surface, we also have

$$
\begin{aligned}
\omega_{12} & =h \omega_{1}, \quad \omega_{13}=a \omega_{1}, \quad \omega_{23}=c \omega_{2}, \\
d h^{\prime} & =\left(1+h^{\prime 2}\right) \omega_{23}, \\
d a & =h \omega_{2}+\lambda \omega_{1}, \quad d c=\mu \omega_{2}
\end{aligned}
$$

where $\lambda$ and $\mu$ are two functions.

Proof II: Secondly we prove that the surface is an $M$-surface. For this purpose we examine its family of lines of curvature, which are defined by the equation

$$
\omega_{1}=0
$$

and are denoted by $\left\{\Gamma_{2}\right\}$. Along any curve $\Gamma_{2}$, we have $\omega_{12}=h \omega_{1}=0$, so that $\Gamma_{2}$ is a geodesic. Letting $\omega_{2}=d s$, we obtain equations for $\Gamma_{2}$,

$$
\begin{aligned}
& \frac{d x}{d s}=e_{2}, \quad \frac{d e_{2}}{d s}=c e_{3}, \\
& \frac{d e_{3}}{d s}=-c e_{2}, \quad \frac{d e_{1}}{d s}=0 .
\end{aligned}
$$


Hence $\Gamma_{2}$ is a plane curve with curvature $c$, and the plane has normal vector $e_{1}$. From (3.23),

$$
d c=\mu \omega_{2} \cong 0\left(\bmod \omega_{2}\right)
$$

meaning that all the curves $\left\{\Gamma_{2}\right\}$ are congruent to each other, because their curvatures are the same.

Since

$$
\begin{aligned}
d e_{1} & =\omega_{12} e_{2}+\omega_{13} e_{3}=\left(h e_{2}+a e_{3}\right) \omega_{1} \\
& =a\left(h^{\prime} e_{2}+e_{3}\right) \omega_{1},
\end{aligned}
$$

the intersection of two neighbouring planes of the curve $\Gamma_{2}$ is a line in the direction

$$
e_{1} \times d e_{1}=a\left(-e_{2}+h^{\prime} e_{3}\right) \omega_{1} .
$$

By (3.21), we have

$$
d\left(-e_{2}+h^{\prime} e_{3}\right)=c h^{\prime}\left(-e_{2}+h^{\prime} e_{3}\right) \omega_{2} .
$$

Hence this direction is fixed. It follows that all planes of the lines $\Gamma_{2}$ of curvature are the tangent planes of a cylinder $Z$. The generator of $Z$ is parallel to the fixed direction. So the surface $M$ is a Molding surface.

We wish to explain geometrical properties of the other family of lines of curvature $\left\{\Gamma_{1}\right\}$, which are defined by the equation

$$
\omega_{2}=0 \text {. }
$$

It is known that all curves $\Gamma_{1}$ are orthogonal trajectories of the tangent planes of $Z$. We have the following lemma concerning this situation.

Lemma. Let $Z$ be a cylinder and let its normal section be a plane curve $C$. Suppose the family of tangent planes of $Z$ is $\left\{\Pi_{s}\right\}, a \leqslant s \leqslant b, a<b, a, b \in \mathbf{R}$. If the orthogonal trajectory is a curve $\Gamma$, then:

(1) $\Gamma$ is a plane curve, and the plane $\Pi$ which includes the curve $\Gamma$ is orthogonal to the direction of the generator of $Z$. In other words, the plane $\Pi$ is orthogonal to a fixed direction.

(2) $\Gamma$ is a involute of intersection $Z \cap \Pi$ of $Z$ and the plane $\Pi$, and the intersection is congruent to a curve $C$. So we can say that $\Gamma$ is an involute of the curve $C$. $Z$ :

Proof: We can choose a frame $O i j k$ or $O x y z$ in $E^{3}$ such that for the cylinder

$$
m(s, z)=m(s)+z k,
$$


where for the curve $C$ :

and

$$
\begin{aligned}
m(s) & =x(s) i+y(s) j, \\
m^{\prime} & =\alpha, \\
\alpha^{\prime} & =\kappa \beta, \quad \beta^{\prime}=-\kappa \alpha, \\
\alpha k & =0, \quad \beta k=0 .
\end{aligned}
$$

The family of the tangent planes of $Z$ is $\left\{\Pi_{s}\right\}$, where

$$
\begin{gathered}
\Pi_{s}: \rho_{s}(u, v)=m(s)+u \alpha(s)+v k, \\
-\infty<u, \quad v<+\infty,
\end{gathered}
$$

where $u, v$ are the parameters of points on the plane $\Pi_{a}$.

Now we can express the orthogonal trajectory $\Gamma$ of the $\left\{\Pi_{A}\right\}$ by

$$
\Gamma: \rho(s)=m(s)+u(s) \alpha(s)+v(s) k .
$$

Taking the derivative of (3.29) using (3.26), we get

$$
\rho^{\prime}=\left(1+u^{\prime}\right) \alpha+\kappa u \beta+v^{\prime} k .
$$

Since $\Gamma$ is the orthogonal trajectory, its tangent line is along the normal of the plane II, , so

$$
\rho^{\prime} \alpha=0, \quad \rho^{\prime} k=0 .
$$

Inserting (3.30) into (3.31), in view of (3.27), we have

$$
1+u^{\prime}=0, \quad v^{\prime}=0 .
$$

From the above equations

$$
u=s_{0}-s, \quad v=v_{0}(=\text { constant }) .
$$

Inserting (3.32) into (3.29), we get

$$
\Gamma: \rho(s)=m(s)+\left(s_{0}-s\right) \alpha(s)+v_{0} k .
$$

It follows that the curve $\Gamma$ is on the plane $\Pi: z=v_{0}$, and it is an involute of the intersection

$$
Z \cap \Pi: \rho(s)=m(s)+v_{0} k .
$$

So we obtain the conclusions (1) and (2) in the lemma.

Using Theorem 2 and the lemma, we get the following Theorem 3. 
THEOREM 3. Two families of lines of curvature on the $M$-surface are:

(1) Second family $\left\{\Gamma_{2}\right\}$; every curve $\Gamma_{2}$ is a plane curve such that pairs of curves are congruent. Its plane is parallel to a fixed direction which is the generator of $Z$.

(2) First family $\left\{\Gamma_{1}\right\}$; every curve $\Gamma_{1}$ is a plane curve such that pairs are not congruent, in general. Its plane is orthogonal to a fixed direction which is the generator of $Z$. Every curve $\Gamma_{1}$ is an involute of the curve $C$.

EXAMPLE. Surfaces of revolution are special examples of Molding surfaces. In this case, the cylinder $Z$ becomes a straight line. The first family of lines of curvature $\left\{\Gamma_{1}\right\}$ is a set of parallels. $\Gamma_{1}$ is a circle, and it is the "involute" of the "point". The second family of lines of curvature $\left\{\Gamma_{2}\right\}$ is a set of meridians. They are congruent to each other.

COROLlary. Let a cylinder $Z$ be given by

$$
\begin{aligned}
& \rho(s, v)=m(s)+v k, \\
& k m=0, \quad \alpha=m^{\prime},
\end{aligned}
$$

and a plane curve $C$ on some tangent plane $\Pi_{0}$ of $Z$, and $C$ be given by

$$
\rho(t)=m\left(s_{0}\right)+u(t) \alpha\left(s_{0}\right)+v(t) k .
$$

Then we have the Molding surface $M$ :

$$
\rho(s, t)=m(s)+\left(s_{0}-s\right) \alpha(s)+u(t) \alpha\left(s_{0}\right)+v(t) k .
$$

\section{4. $L C$-SURFACES OF THIRD TYPE}

Now we study $L C$-surfaces of the third type. From (3.1)-(3.3) we are given

$$
\begin{gathered}
\theta_{1}=k^{\prime} \omega_{13}, \quad \theta_{2}=h^{\prime} \omega_{2 \mathrm{~s}}, \\
\omega_{12}=h^{\prime} \omega_{13}+k^{\prime} \omega_{23} .
\end{gathered}
$$

Let

$$
\sigma=h^{\prime} k^{\prime}
$$

We denote the differentials of $h^{\prime}$ and $k^{\prime}$ by

$$
\begin{aligned}
& d h^{\prime}=\alpha \omega_{13}+\beta \omega_{23} \\
& d k^{\prime}=\gamma \omega_{13}+\delta \omega_{23}
\end{aligned}
$$


so that

$$
\begin{aligned}
d \theta_{1}-2 \theta_{1} \wedge \theta_{2} & =-(\delta+\sigma) * 1 \\
d \theta_{2}-2 \theta_{1} \wedge \theta_{2} & =(\alpha-\sigma) * 1 \\
* 1 & =\omega_{13} \wedge \omega_{23} .
\end{aligned}
$$

From (1.27), using (4.5), we have

$$
\alpha+T \delta=(1-T) \sigma
$$

From the Gaussian equation (3.6), using (4.4), it follows that

$$
\beta-\gamma=2 r
$$

where

$$
2 \tau=h^{\prime 2}+k^{\prime 2}+1
$$

Taking exterior derivatives of (4), we have

$$
\begin{array}{r}
d \alpha \wedge \omega_{13}+d \beta \wedge \omega_{23}+\left(h^{\prime} \alpha+k^{\prime} \beta\right) * 1=0 \\
d \gamma \wedge \omega_{13}+d \delta \wedge \omega_{23}+\left(h^{\prime} \gamma+k^{\prime} \delta\right) * 1=0
\end{array}
$$

so there exist functions $A, B, \ldots, F$ such that

$$
\begin{aligned}
d \alpha & =A \omega_{13}+\left(B+h^{\prime} \alpha\right) \omega_{23} \\
d \beta & =\left(B-k^{\prime} \beta\right) \omega_{13}+C \omega_{23} \\
d \gamma & =D \omega_{13}+\left(E+h^{\prime} \gamma\right) \omega_{23} \\
d \delta & =\left(E-k^{\prime} \delta\right) \omega_{13}+F \omega_{23} .
\end{aligned}
$$

Taking derivatives of (4.3) and (4.8) and using (4.4), we obtain

$$
\begin{aligned}
& d \sigma=\sigma_{1} \omega_{13}+\sigma_{2} \omega_{23}, \\
& \sigma_{1}=h^{\prime} \gamma+k^{\prime} \alpha, \quad \sigma_{2}=h^{\prime} \delta+k^{\prime} \beta, \\
& d \tau=\tau_{1} \omega_{13}+\tau_{2} \omega_{23}, \\
& \tau_{1}=h^{\prime} \alpha+k^{\prime} \gamma, \quad \tau_{2}=h^{\prime} \beta+k^{\prime} \delta .
\end{aligned}
$$

Taking derivatives of (4.6), we get

$$
(\sigma+\delta) d T+T(d \sigma+d \delta)=d \sigma-d \alpha
$$


Using (4.6), (4.10) and (4.11) from (4.12) we have

$$
\begin{gathered}
(\sigma+\delta)^{2} d T+\left[(\sigma-\alpha)\left(E-k^{\prime} \delta+\sigma_{1}\right)+(\sigma+\delta)\left(A-\sigma_{1}\right)\right] \omega_{13} \\
+\left[(\sigma-\alpha)\left(F+\sigma_{2}\right)+(\sigma+\delta)\left(B+k^{\prime} \alpha-\sigma_{2}\right)\right] \omega_{23}=0
\end{gathered}
$$

Inserting (4.6) into (1.26), we have

$$
(\sigma+\delta)^{2} d T-2(\sigma-\alpha)(\alpha+\delta) k^{\prime} \omega_{13}+2(\sigma+\delta)(\alpha+\delta) h^{\prime} \omega_{23}=0 .
$$

Comparing (4.13) and (4.14), we get

$$
\begin{aligned}
& (\sigma+\delta) A+(\sigma-\alpha) E-(\alpha-\sigma)(2 \alpha+\delta) k^{\prime}-(\alpha+\delta) \sigma_{1}=0 \\
& (\sigma+\delta) B+(\sigma-\alpha) F-(\delta+\sigma)(\alpha+2 \delta) h^{\prime}-(\alpha+\delta) \sigma_{2}=0
\end{aligned}
$$

Using (4.10), the above equations become

$$
\begin{aligned}
& (\sigma+\delta) A+(\sigma-\alpha) E=\gamma(\alpha+\delta) h^{\prime}+[\alpha(3 \alpha+2 \delta)-\sigma(2 \alpha+\delta)] k^{\prime} \\
& (\sigma+\delta) B+(\sigma-\alpha) F=[\delta(2 \alpha+3 \delta)+\sigma(\alpha+2 \delta)] h^{\prime}+\beta(\alpha+\delta) k^{\prime}
\end{aligned}
$$

Taking derivatives of (4.7) using (4.9) and (4.11), we have

$$
\begin{aligned}
& B-D=2 \alpha h^{\prime}+(\beta+2 \gamma) k^{\prime}, \\
& C-E=(2 \beta+\gamma) h^{\prime}+2 \delta k^{\prime} .
\end{aligned}
$$

From the above discussion we obtain the following theorem.

TheOREM 4. Let $M$ be a surface with non-zero Gaussian curvature. The necessary and sufficient condition for it to be an $L C$-surface of the third type is that the first and second derivatives of $h^{\prime}$ and $k^{\prime}, \alpha, \beta, \gamma, \delta$ and $A, B, \ldots, F$ satisfy (4.7), (4.15) and (4.16).

\section{REFERENCES}

[1] L. Bryant, Shiing-shen Chern and Phillip A. Griffiths, Exterior Differential Systems: Proceedings of 1980 Beijing DD-Symposium (Science Press, Beijing, China). Vol. 1, pp. 219-338 (Gordon and Breach, New York).

[2] Shiing-shen Chern, 'Deformation of surfaces preserving principal curvatures', Differential Geometry and Complex Analysis (1985), 155-163.

[3] Wenmao Yang, 'Infinitesimal $O$. Bonnet-deformations of surfaces in $E^{3}$ ', Differential Geometry and Topology, Proceedings, Tianjin 1986-87, in Lecture Notes in Math. 1369, pp. 306-321 (Springer-Verlag, New York, 1989).

[4] Wenmao Yang (to appear), On O. Bonnet III - Isometry of Surfaces in $E^{3}$ to appear. 